\title{
Research on the Construction of Smart City Emergency Management System Under Digital Twin Technology: Taking the Practice of New Coronary Pneumonia Joint Prevention and Control as an Example
}

\author{
Tang Zhihong ${ }^{1}$ Peng Shirui ${ }^{2}$ Zhou Xianyong ${ }^{3 *}$
}

\author{
School of Public Affairs and Law, Southwest Jiaotong University, Chengdu, Sichuan 610036, China \\ *Corresponding author. Email: 1481012574@qq.com
}

\begin{abstract}
Under the great tide of smart city construction, digital twin technology combined with modern information means such as big data and cloud computing provides new ideas and methods for urban governance at this stage. In order to explore the solutions to the problems suc $3 \mathrm{~h}$ as information asymmetry, chaotic command and dispatch, and lagging local government decision-making in New Coronary Pneumonia, this paper proposes to introduce a digital twin technology to build the epidemic prevention and control mechanism, which is under the "dual system" coupling mode of smart city twin system and emergency management twin system. It could retrieve epidemic information accurately and timely according to different administrative levels, break the existing central space joint prevention and control mechanism based on physical space. In the construction of the new emergency mechanism, the virtual digital space will no longer be limited to the boundary of the real space, and it can collect epidemic-related information at a second-level speed and accurately implement policies.
\end{abstract}

Keywords: Smart city, digital twin technology, joint defense and joint control emergency mechanism

\section{INTRODUCTION}

On January 23, 2020, the city of Wuhan was closed, and a battle against all epidemics started. In the face of a sudden outbreak, how to cure, how to prevent, and how to control are the three pressing issues before all of us. Unlike SARS, seventeen years ago, the epidemic control was faced with various changes such as closer regional economic cooperation, denser urban populations, more frequent movement of personnel and materials, and faster dissemination of information and public opinion, which brought a huge challenge to epidemic prevention and control. Therefore, building an all-round and efficient emergency joint prevention and control mechanism that matches the modern information society has become an essential part of our modern governance system. Based on the analysis of the current status of new coronary pneumonia prevention and control mechanism, this paper introduces digital twin technology to continuously expand the extension of the joint prevention and control mechanism. In the information age of the Internet of Everything, digital twin technology provides the emergency management system for smart cities. The use of digital twin technology can realize the recording and simulation of the entire life cycle of physical objects, build a complex system of one-to-one correspondence, virtual and real interaction, and achieve the purpose of integrating information and material resources and intelligent collaborative decision-making. The introduction of digital twin technology can solve the problems of information asymmetry and local government decision lag in the prevention and control process, and achieve joint prevention and control of major public health events in smart cities.

\section{LITERATURE REVIEW AND BRIEF EVALUATION}

\subsection{Smart city and digital twin technology}

The concept of smart city covers all aspects of urban development. Italian scholar Papa R. and his colleagues believe that the development of smart city includes three directions: technology center, people-oriented and combination of the two [1]. Dong Hongwei and Kou Yongxia summarized the six cores of smart city connotation: smart technology, smart people, smart system, smart economy, and smart environment [2]. It can be seen that technology is a foundation and important support for smart city construction. The essence of smart city construction is the urban economic and social development and changes in production and lifestyle that guided by modern data and communication technology 
innovations such as big data, cloud computing, artificial intelligence, sensors, and communication.

As one of the latest development directions of modern information technology, digital twin technology can be traced back to the research of the US military in the aerospace system in the 1960s and 1970s. Professor Grieves proposed the concept of digital twin in a narrow sense, he positioned the digital twin as a set of virtual information structures from the micro-atomic level to the macro-geometric level that comprehensively describe potential production or actual manufactured products [3]. As the understanding of this technology continues to deepen, digital twins are broadly defined as technologies, processes, and methods for constructing digital objects that correspond exactly to physical entities. This concept includes three main parts: the physical space entity, the digital model of the virtual space, and the data and information interaction system between the physical entity and the virtual model [4].

After the EU, South Korea, Japan, Germany, Singapore and other countries and regions, China has set off a wave of smart city construction. However, there are problems in smart cities, such as lack of overall planning, serious homogenization between cities, separation of functional systems from reality, and difficulty in resource integration [5]. The application of digital twin technology has injected new vitality into the construction of smart cities. Traditional smart cities mainly focus on information construction, which can improve the efficiency of vertical industries or professions, can also solve the "information island" problem through big data integration technology. But the data twin technology can simulate, predict and infer the final improvement effect of the governance plan in advance through simulation technology, and truly give the city a "smart brain". When major public safety events such as fires, floods, earthquakes, infectious diseases affect the sustainable development of cities, the application of digital twin technology can achieve "one point trigger, multi-party linkage, orderly scheduling, reasonable division of labor, closed-loop feedback"[6].

\subsection{The concept and application of the joint defense}

The joint defense and joint control emergency mechanism is a specific type of government emergency management organization system and belongs to the comprehensive coordination agency under the leadership of the government. In recent years, many scholars have conducted in-depth research on the emergency mechanism of joint defense and joint control. $\mathrm{Hu}$ Yinglian believes that the mechanism mainly involves the cooperation of multiple departments to respond to emergencies that have not yet launched relevant emergency plans. The main responsibilities include discussing the form of judgment at the meeting, studying and determining strategies; agreeing on relevant policies, responding to plans and major measures; coordinating and guiding the implementation of policies, and conducting supervision and inspection [7]. In the health system, Cheng Aijun, Qi Hai propose a relatively complete concept of joint regional prevention and control of diseases: That is, in the prevention and control activities, in order to increase the control of certain major diseases that seriously endanger the health of the people, a work strategy that uses cross-replacement and joint action to implement disease prevention and control measures to prevent and control the spread of related diseases [8]. The core of the joint defense and joint control mechanism for emergency management is the cooperation of multiple departments under the leadership of governments at all levels. Unlike the conventional emergency management agencies based on the bureaucracy. This flat working mechanism can unblock information and improve decision-making efficiency.

Based on scholars' in-depth analysis of the concept, characteristics, and mechanism of digital twin technology, it can be foreseen that the application of this technology in urban management has already begun. However, there is little research on the specific role of digital twin technology in the emergency management process of major security incidents. Therefore, the introduction of digital twin technology into the emergency management process of major security incidents has profound theoretical value and obvious practical significance for making up for the shortcomings of the existing mechanism and further exerting the advantages of the joint defense and joint control working mechanism.

\section{THE WORKING MECHANISM OF THE JOINT PREVENTION AND CONTROL OF THE NEW CORONARY PNEUMONIA}

On January 22, 2020, the central government launched a joint prevention and control mechanism composed of 32 departments led by the National Health Commission. In the ensuing executive meeting of the Political Bureau of the CPC Central Committee, the Party Central Committee established a leading group for epidemic response work to comprehensively strengthen the epidemic prevention and control work in Wuhan, Hubei, and then sent working groups to other provinces to guide local prevention and control work. At the local level, according to the epidemic situation in the region, the joint defense and joint control mechanism from local public security organs, market supervision department to transportation, civil aviation, railways, regional information and other departments has also been launched simultaneously across the country. So far, a relatively complete and flexible joint defense and joint control response mechanism has been established from top to bottom throughout the country. (refer with: Fig. 1): 


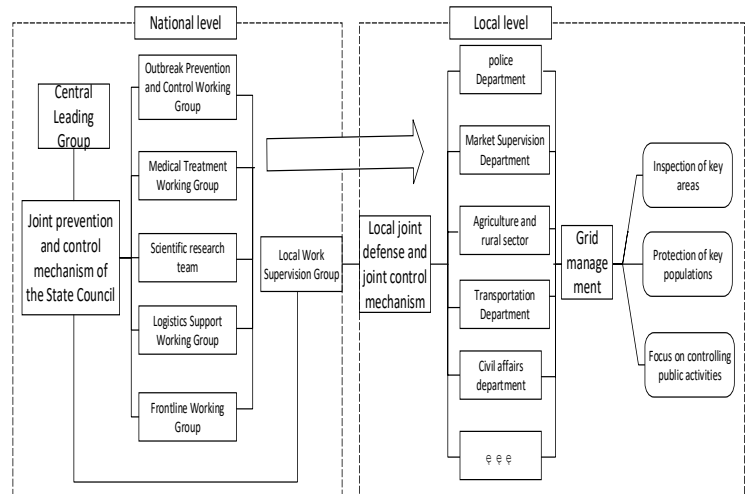

Fig. 1 China's central and local joint prevention and control emergency mechanism

Although the central government showed an agile emergency response capacity during the full outbreak, the Wuhan Municipal Government has been slow to respond for a long period of time in the early stage of the outbreak. The problems of the Chinese government in the prevention and control of the epidemic situation are generally attributed to the two reasons of information asymmetry and local decision-making lag. In fact, there are causal relationships between the two major causes. The root cause of the lag in local government decision-making is still due to the asymmetry of the information, which leads to the mismatch of measures. If the digital twin technology is used to construct an epidemic prevention and control digital twin model, it can not only solve the problems of information collection, collation and feedback in the joint prevention and control mechanism of our country, but also further improve the existing emergency mechanism, the local government's lack of investment in disaster prevention and control, and comprehensively improve the local government's emergency management and control capabilities

\section{INITIAL CONSTRUCTION OF SMART CITY EMERGENCY MECHANISM INTRODUCING DIGITAL TWIN TECHNOLOGY}

The city has a dense crowd and huge resources. It is a large warehouse and market where large amounts of data such as documents, pictures, audio, video, geographic location, and social networks are generated. It is the main position and main battlefield for using massive data such as population, transportation, weather, housing, fire protection, environmental protection. To a certain extent, the quality of urban public safety depends on the quality of the city 's data and its external environmental information processing [9]. Urban emergency management is a part of comprehensive urban management. The construction of the joint prevention and control mechanism for epidemic prevention requires the city as the space carrier for the occurrence of incidents. Correspondingly, the construction of the joint prevention and control mechanism under the digital twin technology must use the virtual urban space scene as the carrier, on this basis, combined with a specific scenario of emergency management of an emergency. Therefore, the construction of joint prevention and control mechanism under digital twin technology should be a combination of digital twin city construction and digital twin emergency management construction.

\subsection{The establishment of central and local joint defense and emergency control mechanism under digital twin technology}

Digital twin city is a new presentation of digital twin technology applied to smart city. Its construction includes two aspects: on the one hand, digital twin technology needs to build a global perception relative to urban infrastructure, including static perception of urban roads, schools, hospitals, shopping malls, and dynamic perception of traffic flow, pedestrian flow, energy consumption, equipment operation, climate change, and the handling of urban emergency management is based on the construction of various scenarios. On the other hand, the service object of urban governance is people, especially in the period of emergencies, managers need to control the movement of urban residents in a timely manner, accurately issue command decisions to stabilize people's hearts. Therefore, the construction of smart cities must also be oriented to urban residents. Digital twin technology can form a user portrait of each city resident, using sensors such as smartphones and smart terminals held by people to comprehensively collect and organize personal basic information, global coverage monitoring information and ubiquitous perception information. Eventually, the individual in the city can be tracked for the entire period and entire process, and timely presents the individual's trajectory, social activities, and behavior in a digital twin scene. Based on the construction of digital twin cities, the digital twin epidemic prevention and control mechanism is mainly composed of the following parts. (refer with: Fig. 2):

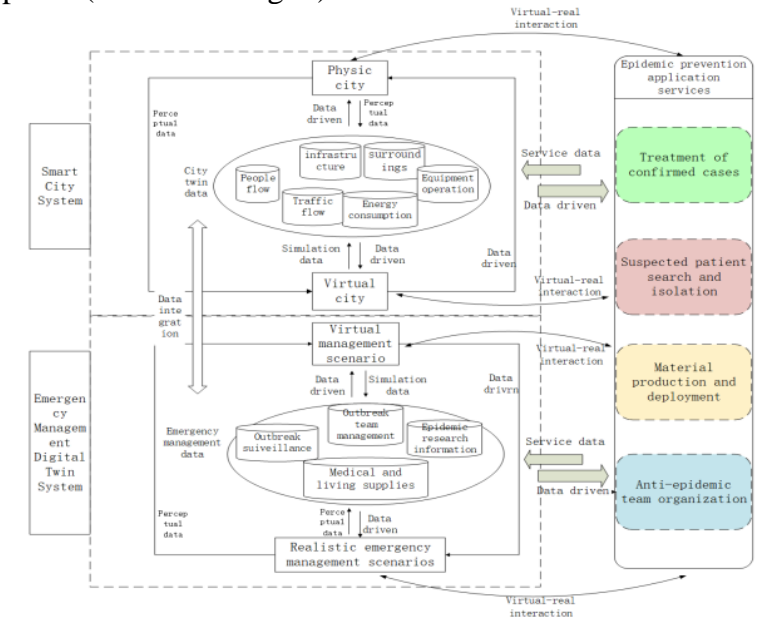

Fig. 2 Construction of smart city epidemic prevention and control mechanism under digital twin technology 


\subsubsection{Physical cities and real emergency management scenarios:}

physical cities are the real cities where urban residents exist. Realistic emergency management scenarios can be understood as real-time scenarios of epidemic occurrence, prevention and control, such as the treatment of infected people, the work of medical staff, production and distribution of epidemic materials.

\subsubsection{Virtual city and virtual emergency management scenario:}

Virtual city is a model corresponding to physical city constructed by digital modeling, which can simulate everything that actually happened in physical city, including the dynamics of buildings, residents, transportation, environment and so on. The virtual emergency management scene as a specific event will also appear in the virtual city model.

\subsubsection{Urban Big Data:}

Urban roads, traffic, river information, virtual city simulations, and various smart city service records collected using network devices such as monitoring and mobile phones are aggregated into urban big data.

\subsubsection{Emergency management twin data:}

The data involved in epidemic prevention work includes not only basic urban big data, but also real-time control of medical and basic living materials information, epidemic situation monitoring information, epidemic situation management team information, and epidemic situation research information.

\subsubsection{Virtual-Real Interaction:}

The virtual-real interaction connects the physical world with the digital world. Various information occurring in the physical space can be searched in the virtual space and fed back in time. The personnel can more accurately, timely and objectively grasp the state of the object and further enhance the coupling with the physical object.

\subsubsection{Epidemic prevention and control application services:}

Based on the construction of smart cities, the joint prevention and control of the epidemic can be comprehensively and accurately realized relying on digital twin technology. It is also used in medical treatment of confirmed cases, search and isolation of suspected patients, deployment of supplies, and organization of epidemic prevention teams service.

Driven by the digital twin technology, urban governance will change to the direction of intelligent and autonomous operation, will no longer be limited by the size of the grid and the boundary of the physical space. The urban grid governance model will develop toward de-grid [10]. At this time in the epidemic prevention and control, the central government, local governments at all levels, and grassroots street communities can rely on digital twin technology to establish epidemic prevention and control systems in various administrative regions, and build a set of virtual information network from bottom to top. Decision makers can retrieve the epidemic prevention and control data in the corresponding area timely according to the size of their responsibilities and rights, breaking the original epidemic information reporting mechanism. The system can quickly find problems, capture key information and make decisions. Thus, a new type of central and joint defense and emergency control mechanism under digital twin technology has been established. (refer with: Fig. 3)

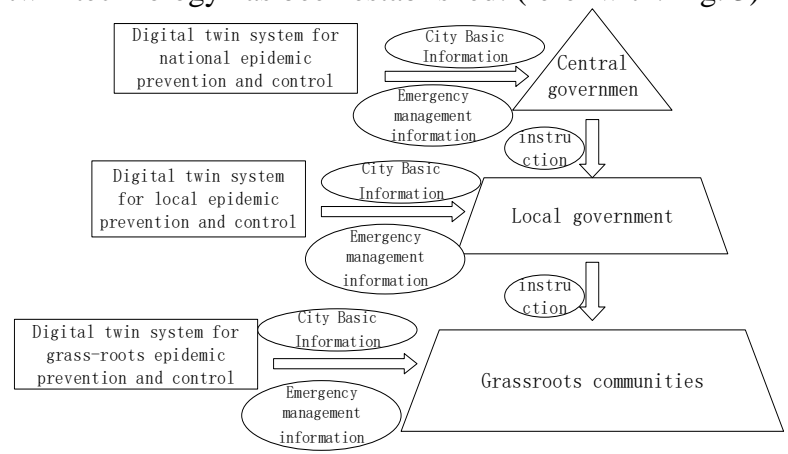

Fig. 3 Preliminary construction of smart city emergency management system under data twin technology

\subsection{The key points of the construction of the joint defense and joint control emergency mechanism under the digital twin technology}

Based on the prevention and treatment of New Coronary Pneumonia, digital twin technology has given new inspiration to the joint prevention and control of the epidemic. However, in addition to the technical difficulties, there are also many challenges to transform ideas and theories into applications:

\subsubsection{Regional construction balance:}

the traditional joint defense and joint control mechanism is established by administrative means, and the digital twinbased joint defense and joint control mechanism is built on a relatively complete virtual information space, which requires a considerable technical foundation and covers the construction of a series of technical activities including support systems, data management, dynamic modeling, data calculation and targeted services, and iterative 
optimization. China has 31 provinces, autonomous regions and municipalities. The social and economic development of each region varies greatly, and their technological innovation capabilities are also different. This will objectively cause the asynchronous construction of the virtual city, which will affect the use of digital twin technology to build a nationwide emergency management mechanism.

\subsubsection{Integration of digital administration:}

The new coronary pneumonia epidemic, as a public affairs with obvious external characteristics, requires cross-border actions by local governments. However, the regional administrative boundary is the absolute spatial boundary for the local government to exercise public power. No local government has the right to cross-border law enforcement unless it is licensed by a superior government. The data model constructed by the digital twin technology covers all the information in the region, The local government is also reluctant to share with each other because of local protectionism. Once the central government does not control the national information in time, the best timing for prevention and control will be delayed. Therefore, the use of digital twin technology to build an emergency system is necessary to achieve integrated joint prevention and control of digital space.

\subsubsection{Data collection and opening:}

To build a digital twin model that accurately reflects the current dynamics, it is necessary to collect the original data in the early stage. At present, most of the data in China is in the hands of the official, but the market and society are more creative and professional than the government, can more accurately grasp the demand. Therefore, we need to think about how to take measures to give full play to the data collection role of the market and society.

\subsubsection{Data security and humanized governance:}

In the era of big data, various mobile phone applications can grasp information such as our action trajectory, consumption habits, geographic positioning, and home address, that initially applied the concept of digital twin technology, but most of them Consumers reject such data tracking and worry about security issues caused by privacy leaks. In the future, the digital twin technology's fullprocess, full-time and panoramic recording of the physical world will inevitably make everything completely "transparent", which will cause residents' panic to a certain extent. In addition to solving the problem of data security, it is also necessary to think about how to strike a balance between standardized governance and humanized governance.

\section{CONCLUSION}

This article puts forward the preliminary ideas and suggestions for the construction of smart city emergency management system under digital twin technology, that is, to use the virtual urban space scene as a carrier, combined with a specific scene of an emergency, to build an urban twin system and an emergency management twin system. The decision maker then retrieves the epidemic prevention and control data of the corresponding area in time according to the size of the responsibilities and rights. However, as an advanced technology and method, digital twin technology is still at the initial stage of innovation, and it still has a long way to go before widespread adoption, and how to integrate this technology with rapidly developing information technology is also the focus of the next stage.

\section{REFERENCES}

[1] Papa R, Gargiulo C, Galderis A. Toward an Urban Planners' Perspective on Smart City[J]. Journal of Land Use, Mobility and Environment, 2013, 1: 5-17.

[2] Dong Hongwei, Kou Yongxia. Criticism and Practice of Smart City_ Overseas Literature Review [J]. Urban Planning, 2014,38 (11): 52-58.

[3] Dai Sheng, Zhao Gang, Yu Yong, Wang Wei: "Digital Product Definition Development Trend: From Prototype to Twins", "Journal of Computer-Aided Design and Graphics", No. 8, 2018, pages 1554-1558.

[4] Chen Cai: "Concept and Features of Digital Twin Cities", People's Posts and Telecommunications, 6th Edition, December 15, 2017.

[5] Chao Liang: "Problems and Corrections in the Construction of Smart Cities in China", "House", Issue 6, 2019.

[6] Zhou Yu, Liu Chuncheng: "Logic and Innovation of Building a Digital Twin City in Xiong'an New District", "Urban Development Research" Volume 25, Issue 10, 2018.

[7] Hu Yinglian: "Comparative Research on China's Emergency Management Organization System-Taking Public Health Emergencies as an Example", Journal of University of Science and Technology Beijing (Social Science Edition) 2012 Vol. 28 No. 2, pp. 137-142.

[8] Cheng Aijun, Qi Hai, Zuo Guifeng, Shen Yuexia: "Regional Joint Prevention and Control of Major Diseases", Journal of Preventive Medicine Information, 2007, Issue 1, pages 90-92. 
[9] He Zhen, Zhou Fangjian, Yang Wen: "Research on the Collaborative System Innovation of Urban Emergency Management Industry in the Era of Big Data", "Journal of Xiangtan University (Philosophy and Social Sciences Edition)", Volume 40, Issue 6, 2016, pages 26-31.

[10] Zhang Yuxiong: "Urban Governance under the Digital Wave: From Fine to Intelligent", "Telecommunication Network Technology", No. 3, 2018, pages $9-11$. 